\title{
New Efficient Synthesis of 3-Carboxylquinolines
}

\author{
S. Kirankumar, D. Rambabu, N. Chandra Sekhar ${ }^{\dagger}$, ASG. Prasad ${ }^{\dagger}$, and M. V. Basaveswara Rao ${ }^{\dagger, *}$ \\ Department of Chemistry, K L University, Vaddeswaram Guntur-522 502, A. P, India \\ ${ }^{\dagger}$ Department of chemistry, Krishna University, Machilipatnam-521 001, A. P, India. \\ *E-mail: professormandava@gmail.com
}

(Received January 20, 2012; Accepted April 17, 2012)

\begin{abstract}
Rapid and efficient synthesis of substituted 3-carboxylquinoline derivatives from 4-chloro-3-formylcoumarin and substituted anilines using $30 \% \mathrm{H}_{2} \mathrm{SO}_{4}$ in methanol at room temperature within the duration of 5-30 min., through domino condensation-cyclization-ring opening reaction.
\end{abstract}

Key words: 3-Carboxylquinoline derivatives, 4-Chloro-3-formylcoumarin, Anilines

\section{INTRODUCTION}

Functionalized quinoline scaffolds obtained from natural sources and their synthetic analogs are of considerable importance due to their wide spectrum of biological profiles. Quinoline derivatives proved to be possessing antimalarial, ${ }^{1}$ anti-inflammatory, ${ }^{2}$ anticancer, ${ }^{3}$ antibiotic, ${ }^{4}$ antihypertensive, ${ }^{5}$ tyrokinase PDGF-RTK inhibiting agents, ${ }^{6}$ anti HIV, ${ }^{7}$ DNA binding capability, ${ }^{8}$ antitumor activities, ${ }^{9}$ and DNA-intercalating carrier ${ }^{10}$ activities. In addition to the medicinal significance, multi-substituted quinolines are valuable synthons exploited for the preparation of nanoand mesostructures with enhanced electronic and photonic properties. ${ }^{11}$

The synthesis of quinoline derivatives thus continues to be an attractive area of research, ${ }^{12}$ and the synthesis of various substituted quinolines has been largely described in the literature through different strategies. ${ }^{13,14}$ For example, preparation of quinolines by Lewis acid catalyzed cyclization of 1,5-diaryl-1,5-diazapentadiene salts have been reported in $1923 .{ }^{15}$ These compounds were prepared by the action of primary arylamines on $\beta$-chlorovinylaldehydes at room temperature. ${ }^{16,17}$ Some diazapentadiene salts undergo intramolecular cyclization when heated under reflux in acetic acid or in alcohols of similar boiling point and are converted into quinolines. ${ }^{18}$ The mechanism under these conditions has been rationalized as an electrocyclic ring closure with elimination. ${ }^{19}$ Similarly the thermolysis of diazapentadiene analogues gives quinolines. ${ }^{20}$ As a consequence, the development of general methods for the synthesis and biological evaluation of new agents, retaining the 'core' quinoline moiety has been the subject of considerable synthetic effort. An essential component of the search for new leads in the drug designing program is the synthesis of molecules, which are novel yet resemble known biologically active molecules by virtue of the presence of some critical structural features. Certain small heterocyclic molecules act as highly functional scaffolds and are known pharmacophores of a number of biologically active and medicinally useful molecules. ${ }^{21}$ The SkraupDoebner-Von Miller quinoline synthesis, which generally refers to the reaction of $\alpha, \beta$-unsaturated carbonyl compounds with anilines to give quinolines, has been of great value for constructing the quinoline system since its discovery one and a quarter centuries ago. ${ }^{22}$

A reversal of the standard regiochemistry of the SkraupDoebner-Von Miller quinoline synthesis was observed when anilines were condensed with $\gamma$-aryl- $\beta, \gamma$-unsaturated $\alpha$-ketoesters in refluxing TFA. The reaction is proposed to involve 1,2-addition of the anilines to $\gamma$-aryl- $\beta, \gamma$ unsaturated $\alpha$-ketoesters to form Schiff's base adducts, followed by cyclization and oxidation. The products were shown to the 2-carboxy-4-arylquinolines by spectroscopy and X-ray crystallographic analysis. ${ }^{23}$ Similarly, a variety of substituted quinolines are synthesized from imines and enolizable carbonyl compounds under aerobic conditions, in DMSO, and a catalytic amount of $\mathrm{HCl}$ activates carbonyl compounds to give the quinolines. ${ }^{24}$ Dieter Heber reported the reaction of 4-chloro-3-formylcoumarin with p-substituted anilines to yield 6-Oxo-6H-[1] benzopyrano[4,3-b] quinoline derivatives. ${ }^{25}$

However, most of the synthetic routes suffer from various problems: (1) harsh conditions, ${ }^{26}(2)$ multisteps, ${ }^{27}$ and (3) a large amount of promoters such as a base, ${ }^{28}$ expensive and/or harmful metals, ${ }^{29}$ the oxidants for the aromatization, ${ }^{30}$ and other additives. ${ }^{31}$ Thus, the development 


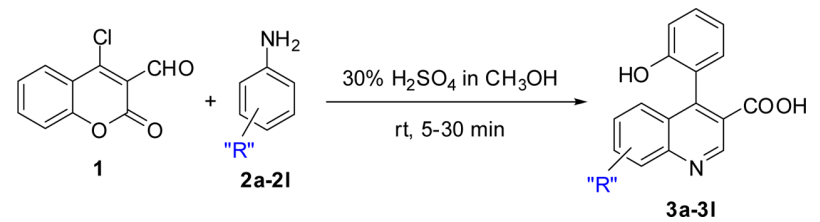

Scheme 1. Synthesis of 3-carboxyl quinolines 3a-31.

of simple and practical methods for synthesizing functionally substituted quinolones is of current interest. ${ }^{32-33}$ So thrust in framing and developing synthetic methodologies for new and highly functionalized quinoline moieties is of current interest.

\section{RESULTS AND DISCUSSION}

In this context herein, we are reporting one pot, acidcatalyzed, new methodology for the fast and efficient synthesis of highly functionalized quinoline derivatives. Reaction of acid-catalyzed $\left(30 \% \mathrm{H}_{2} \mathrm{SO}_{4}\right.$ in methanol $) 4-$ chloro-3-formylcoumarin (1) with substituted anilines (2a-2l) gave the desired substituted 3-carboxylquinolines (3a-3l) at room temperature in the duration of within 5-30 min (Scheme 1, Table 1). The reaction sequences employed for the synthesis of substituted 3-carboxylquinoline derivatives are shown in Scheme 1. The reaction proceeds through the domino Schiff base condensation followed by cyclization-aromatization-ring-opening as shown in Scheme 2.

\section{CONCLUSIONS}

In conclusion this methodology provides a fast and efficient synthesis of new substituted 3-carboxyquinoline derivatives, which can diversity oriented for the creation of library of these molecules.

\section{EXPERIMENTAL SECTION}

\section{General}

Reactions were monitored by thin layer chromatography (TLC) on silica gel plates ( $60 \mathrm{~F} 254$ ), visualizing with ultraviolet light or iodine spray. ${ }^{1} \mathrm{HNMR}$ and ${ }^{13} \mathrm{CNMR}$ spectra were determined in $\mathrm{CDCl}_{3}$ or DMSO- $d_{6}$ solution by using 400 and $100 \mathrm{MHz}$ spectrometers, respectively. Proton chemical shifts $(\delta)$ are relative to tetramethylsilane (TMS, $\delta=0.00$ ) as internal standard and expressed in ppm. Spin multiplicities are given as s (singlet), d (doublet), $\mathrm{t}$ (triplet) and $\mathrm{m}$ (multiplet) as well as b (broad). Coupling constants $(J)$ are given in hertz. Infrared spectra were recorded on a FT-IR spectrometer. Melting points were determined using melting point apparatus and are uncorrected. MS spectra were obtained on a mass spectrometer. All chemicals and reagents were purchased from commercial sources and purified before use.

\section{General procedure for the Synthesis of 3-carboxyl Quinolines (3a-31)}

A mixture of 4-Chloro-3-formylcoumarin (1) (1.0 mmol), $30 \% \mathrm{H}_{2} \mathrm{SO}_{4}$ in methanol and anilines (2a-2l) (1.0 m.mol) were stirred at room temperature for 5-30 min. (Scheme 1, Table 1). After completion of the reaction, the solid obtained (3a-31) was filtered and washed with methanol. All the compounds (3a-3l) were characterized by their NMR, IR and Mass spectral data.

4-(2-Hydroxyphenyl)quinoline-3-carboxylic acid (3a)

White solid; ${ }^{1} \mathrm{H}$ NMR (400 MHz, DMSO- $d_{6}$ ): $\delta 11.5$ (s, $1 \mathrm{H}), 10.05$ (s, 1H), 9.25 (s, 1H), 8.02 (d, $J=8.4 \mathrm{~Hz}, 1 \mathrm{H})$, $7.65(\mathrm{~d}, J=8.4 \mathrm{~Hz}, 1 \mathrm{H}), 7.55(\mathrm{dd}, J=8.0 \mathrm{~Hz}, J=2.4 \mathrm{~Hz}$, $1 \mathrm{H}), 7.36(\mathrm{dd}, J=8.4 \mathrm{~Hz}, J=2.4 \mathrm{~Hz}, 1 \mathrm{H}), 7.26(\mathrm{~d}, J=7.6$ $\mathrm{Hz}, 1 \mathrm{H}), 7.02-7.09(\mathrm{~m}, 1 \mathrm{H}), 6.84-6.9(\mathrm{~m}, 1 \mathrm{H}), 6.71(\mathrm{~d}, J=$ $8.0 \mathrm{~Hz}, 1 \mathrm{H}) ;{ }^{13} \mathrm{C}$ NMR (100 MHz, DMSO- $\left.d_{6}\right): \delta 168.9$, $154.3,148.2,147.1,140.6,132.4,130.5,129.8,128.8$, $128.5,128.1,127.2,122.2,121.6,121.1,115.8$; IR (KBr) $v_{\max } 3274,3204,3071,2958,1644,1483,1367,1224$, 1145; EI-MS: m/z $264.1(\mathrm{M}-\mathrm{H})^{+}$.

4-(2-Hydroxyphenyl)-6-methylquinoline-3-carboxylic acid (3b)

Off White solid; ${ }^{1} \mathrm{H}$ NMR (400 MHz, DMSO- $\left.d_{6}\right): \delta 11.4$ (s, 1H), $10.01(\mathrm{~s}, 1 \mathrm{H}), 9.21(\mathrm{~s}, 1 \mathrm{H}), 7.92(\mathrm{~d}, J=8.0 \mathrm{~Hz}$, $1 \mathrm{H}), 7.52$ (s, 1H), 7.45 (d, $J=8.0 \mathrm{~Hz}, 1 \mathrm{H}), 7.27$ (d, $J=7.6$ $\mathrm{Hz}, 1 \mathrm{H}), 7.01-7.08$ (m, 1H), 6.83-6.90 (m, 1H), 6.72 (d, $J$ $=8.0 \mathrm{~Hz}, 1 \mathrm{H}), 2.35(\mathrm{~s}, 3 \mathrm{H}) ;{ }^{13} \mathrm{C} \mathrm{NMR}(100 \mathrm{MHz}, \mathrm{DMSO}-$ $\left.d_{6}\right): \delta 169.5,154.8,147.2,146.9,140.2,137.8,134.2$, $129.1,128.9,128.2,127.9,127.2,122.4,121.3,121.1$, 116.8, 25.1; IR (KBr) $v_{\max } 3276,3208,3074,2960,1646$, 1480, 1366, 1220, 1145; EI-MS: m/z $278.1(\mathrm{M}-\mathrm{H})^{+}$.

6-Chloro-4-(2-hydroxyphenyl)quinoline-3-carboxylic acid (3c)

White solid; ${ }^{1} \mathrm{H}$ NMR (400 MHz, DMSO- $d_{6}$ ): $\delta 11.5$ (s, $1 \mathrm{H}), 10.02(\mathrm{~s}, 1 \mathrm{H}), 9.27$ (s, 1H), 7.95 (d, $J=8.4 \mathrm{~Hz}, 1 \mathrm{H})$, $7.72(\mathrm{~s}, 1 \mathrm{H}), 7.61(\mathrm{~d}, J=8.4 \mathrm{~Hz}, 1 \mathrm{H}), 7.32(\mathrm{~d}, J=7.6 \mathrm{~Hz}$, $1 \mathrm{H}), 7.03-7.11(\mathrm{~m}, 1 \mathrm{H}), 6.85-6.92(\mathrm{~m}, 1 \mathrm{H}), 6.80(\mathrm{~d}, J=8.0$ $\mathrm{Hz}, 1 \mathrm{H}) ;{ }^{13} \mathrm{C}$ NMR (100 MHz, DMSO-d $): \delta 169.1,155.3$, $148.4,147.1,139.8,134.4,133.5,131.1,130.3,129.1$, $128.9,127.5,122.5,122.1,121.9,116.8$; IR (KBr) $v_{\max }$ 3272, 3206, 3070, 2956, 1646, 1484, 1367, 1224, 1145; EI-MS: m/z $298.5(\mathrm{M}-\mathrm{H})^{+}$.

4-(2-Hydroxyphenyl)-6-nitroquinoline-3-carboxylic
acid (3d)
$\quad$ Yellow solid; ${ }^{1} \mathrm{H}$ NMR $\left(400 \mathrm{MHz}\right.$ DMSO- $\left.d_{6}\right): \delta 11.5$ 
Table 1. 3-Carboxyl quinoline derivatives 3a-3l produced via Scheme 1

\begin{tabular}{|c|c|c|c|c|c|}
\hline Entry & Anilines & Quinoline Product & Time (min) & $\mathrm{Mp}\left({ }^{\circ} \mathrm{C}\right)$ & $(\%)$ Yield $^{\mathrm{a}}$ \\
\hline 1 & & & 30 & $170-172$ & 98 \\
\hline 2 & $2 b$ & & 20 & $153-154$ & 98 \\
\hline 3 & 20 & & 5 & $173-175$ & 97 \\
\hline 4 & & & 5 & $184-186$ & 99 \\
\hline 5 & $2 e$ & & 15 & $180-182$ & 98 \\
\hline 6 & $2 f$ & & 15 & $186-188$ & 96 \\
\hline 7 & & & 30 & $120-122$ & 95 \\
\hline 8 & & & 15 & $151-153$ & 97 \\
\hline 9 & & & 5 & $136-138$ & 98 \\
\hline 10 & & & 5 & $179-181$ & 99 \\
\hline 11 & $2 \mathrm{k}$ & & 5 & $173-175$ & 98 \\
\hline 12 & & & 5 & $177-179$ & 99 \\
\hline
\end{tabular}

asolated yields. All products (3a-3l) were characterized by NMR, IR and mass spectral data

(s, 1H), $10.03(\mathrm{~s}, 1 \mathrm{H}), 9.65(\mathrm{~s}, 1 \mathrm{H}), 8.71(\mathrm{~s}, 1 \mathrm{H}), 8.49(\mathrm{~d}, J$ $=8.8 \mathrm{~Hz}, 1 \mathrm{H}), 8.31(\mathrm{~d}, J=8.8 \mathrm{~Hz}, 1 \mathrm{H}), 7.35(\mathrm{~d}, J=7.6 \mathrm{~Hz}$,
$1 \mathrm{H}), 7.04-7.12(\mathrm{~m}, 1 \mathrm{H}), 6.86-6.93(\mathrm{~m}, 1 \mathrm{H}), 6.83(\mathrm{~d}, J=8.0$ $\mathrm{Hz}, 1 \mathrm{H}) ;{ }^{13} \mathrm{C}$ NMR $\left(100 \mathrm{MHz}, \mathrm{DMSO}-d_{6}\right): \delta 169.4,155.5$, 


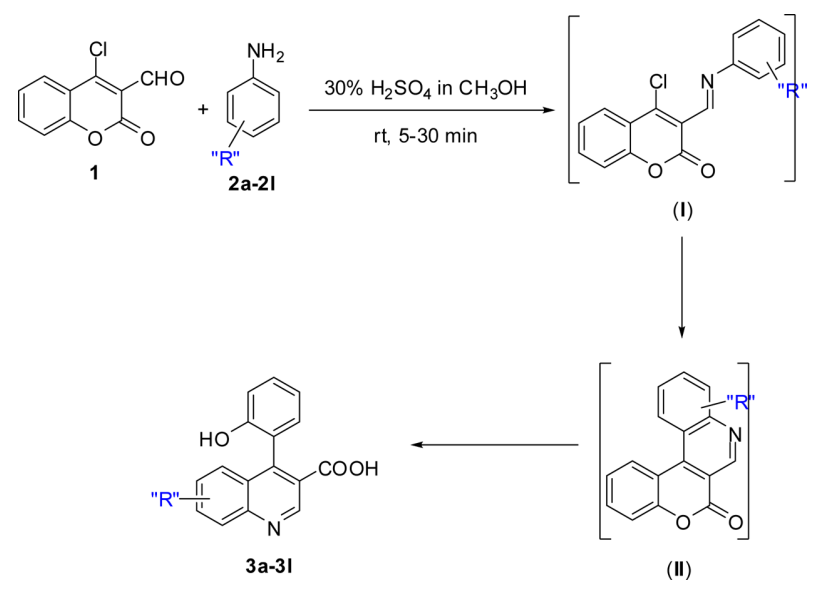

Scheme 2. Proposed reaction mechanism for new quinolines 3a-31.

$152.1,151.5,147.6,143.3,131.2,130.5,129.2,128.9,126.2$, 125.3, 123.7, 122.1, 120.6, 116.9; IR (KBr) $v_{\max } 3274$, 3206, 3074, 2958, 1644, 1483, 1367, 1224, 1145; EI-MS: $\mathrm{m} / \mathrm{z} 309.1(\mathrm{M}-\mathrm{H})^{+}$.

6-Bromo-4-(2-hydroxyphenyl)quinoline-3-carboxylic acid (3e)

Red solid; ${ }^{1} \mathrm{H}$ NMR (400 MHz, DMSO- $d_{6}$ ): $\delta 11.5$ (s, 1H), 10.02 (s, 1H), 9.27 (s, 1H), 8.01 (d, $J=8.4 \mathrm{~Hz}, 1 \mathrm{H})$, $7.97(\mathrm{~s}, 1 \mathrm{H}), 7.77(\mathrm{~d}, J=8.4 \mathrm{~Hz}, 1 \mathrm{H}), 7.32(\mathrm{~d}, J=7.6 \mathrm{~Hz}$, 1H), 7.03-7.11 (m, 1H), 6.85-6.92 (m, 1H), $6.81(\mathrm{~d}, J=8.0$ $\mathrm{Hz}, 1 \mathrm{H}) ;{ }^{13} \mathrm{C}$ NMR (100 MHz, DMSO-d $): \delta 169.3,155.3$, $150.1,148.3,140.2,136.3,131.2,130.9,130.5,129.1$, 128.9, 122.6, 122.2, 122.1, 119.9, 116.7; IR (KBr) $v_{\max }$ 3280, 3208, 3074, 2958, 1648, 1485, 1367, 1224, 1150; EI-MS: m/z $343.0(\mathrm{M}-\mathrm{H})^{+}$.

6-Fluoro-4-(2-hydroxyphenyl)quinoline-3-carboxylic acid (3f)

White solid; ${ }^{1} \mathrm{H}$ NMR (400 MHz, DMSO- $d_{6}$ ): $\delta 11.4$ (s, $1 \mathrm{H}), 10.01(\mathrm{~s}, 1 \mathrm{H}), 9.31(\mathrm{~s}, 1 \mathrm{H}), 8.15(\mathrm{~d}, J=8.4 \mathrm{~Hz}, 1 \mathrm{H})$, $7.46(\mathrm{~d}, J=7.6 \mathrm{~Hz}, 1 \mathrm{H}), 7.36(\mathrm{dd}, J=8.0 \mathrm{~Hz}, J=1.6 \mathrm{~Hz}$, $1 \mathrm{H}), 7.32$ (d, $J=7.6 \mathrm{~Hz}, 1 \mathrm{H}), 7.01-7.09(\mathrm{~m}, 1 \mathrm{H}), 6.83-$ $6.91(\mathrm{~m}, 1 \mathrm{H}), 6.77(\mathrm{~d}, J=8.0 \mathrm{~Hz}, 1 \mathrm{H}) ;{ }^{13} \mathrm{C}$ NMR $(100$ MHz, DMSO- $\left.d_{6}\right): \delta 169.2,162.1,155.4,147.5,146.0,140.5$, 131.6, 130.9, 129.1, 128.9, 122.5, 122.3, 122.1, 121.9, 111.5, 116.8; IR (KBr) $v_{\max } 3276,3210,3074,2960,1644$, 1483, 1367, 1224, 1145; EI-MS: m/z $282.1(\mathrm{M}-\mathrm{H})^{+}$.

7-Bromo-4-(2-hydroxyphenyl)quinoline-3-carboxylic acid (3g)

White solid; ${ }^{1} \mathrm{H}$ NMR (400 MHz, DMSO- $\left.d_{6}\right): \delta 11.5$ (s, $1 \mathrm{H}), 10.02(\mathrm{~s}, 1 \mathrm{H}), 9.41(\mathrm{~s}, 1 \mathrm{H}), 8.31(\mathrm{~s}, 1 \mathrm{H}), 7.61$ (d, $J=$ $8.4 \mathrm{~Hz}, 1 \mathrm{H}), 7.66(\mathrm{~d}, J=8.4 \mathrm{~Hz}, 1 \mathrm{H}), 7.32(\mathrm{~d}, J=7.6 \mathrm{~Hz}$, $1 \mathrm{H}), 7.03-7.11(\mathrm{~m}, 1 \mathrm{H}), 6.85-6.92(\mathrm{~m}, 1 \mathrm{H}), 6.81(\mathrm{~d}, J=8.0$ $\mathrm{Hz}, 1 \mathrm{H}) ;{ }^{13} \mathrm{C}$ NMR (100 MHz, DMSO-d $): \delta 169.3,155.3$,
$149.2,147.3,140.4,132.3,131.8,130.9,130.6,129.1$, $128.9,126.7,122.3,122.0,121.5,116.5$; IR (KBr) $v_{\max }$ 3278, 3206, 3076, 2958, 1648, 1483, 1367, 1224, 1145; EI-MS: m/z $343.0(\mathrm{M}-\mathrm{H})^{+}$.

6,7-Difluoro-4-(2-hydroxyphenyl)quinoline-3-carboxylic acid (3h)

White solid; ${ }^{1} \mathrm{H}$ NMR (400 MHz, DMSO- $\left.d_{6}\right): \delta 11.4$ (s, 1H), 10.02 (s, 1H), 9.33 (s, 1H), 7.76 (d, $J=8.8 \mathrm{~Hz}, 1 \mathrm{H})$, $7.39(\mathrm{~d}, J=8.4 \mathrm{~Hz}, 1 \mathrm{H}), 7.32$ (d, $J=7.6 \mathrm{~Hz}, 1 \mathrm{H}), 7.01$ 7.09 (m, 1H), 6.85-6.91 (m, 1H), 6.78 (d, $J=8.0 \mathrm{~Hz}, 1 \mathrm{H})$; ${ }^{13} \mathrm{C}$ NMR (100 MHz, DMSO- $\left.d_{6}\right): \delta 169.3,157.2,155.8$, 155.3, 148.7, 147.1, 140.5, 130.9, 129.1, 128.9, 122.8, 122.2, $119.1,116.9,114.5,113.8$; IR (KBr) $v_{\max } 3274,3204$, 3071, 2958, 1644, 1483, 1367, 1224, 1145; EI-MS: m/z $300(\mathrm{M}-\mathrm{H})^{+}$.

7,8-Difluoro-4-(2-hydroxyphenyl)quinoline-3-carboxylic acid (3i)

White solid; ${ }^{1} \mathrm{H}$ NMR (400 MHz, DMSO- $d_{6}$ ): $\delta 11.4$ (s, $1 \mathrm{H}), 10.02(\mathrm{~s}, 1 \mathrm{H}), 9.35$ (s, 1H), 7.47 (d, $J=8.0 \mathrm{~Hz}, 1 \mathrm{H})$, $7.32(\mathrm{~d}, J=7.6 \mathrm{~Hz}, 1 \mathrm{H}), 7.17(\mathrm{dd}, J=8.4 \mathrm{~Hz}, J=1.6 \mathrm{~Hz}$, 1H), 7.02- 7.09 (m, 1H), 6.84-6.91 (m, 1H), 6.79 (d, $J=$ $8.0 \mathrm{~Hz}, 1 \mathrm{H}) ;{ }^{13} \mathrm{C}$ NMR $\left(100 \mathrm{MHz}, \mathrm{DMSO}-d_{6}\right): \delta 169.3$, $155.7,151.3,150.2,144.1,141.9,141.3,140.7,130.9$, 129.1, 128.9, 128.3, 122.2, 121.9, 118.5, 116.5; IR (KBr) $v_{\max } 3278,3208,3074,2960,1644,1483,1367,1226$, 1148; EI-MS: m/z $300(\mathrm{M}-\mathrm{H})^{+}$.

4-(2-Hydroxyphenyl)-8-methyl-7-nitroquinoline-3carboxylic acid (3j)

Yellow solid; ${ }^{1} \mathrm{H}$ NMR (400 MHz, DMSO- $d_{6}$ ): $\delta 11.5$ (s, $1 \mathrm{H}), 10.03$ (s, 1H), 9.65 (s, 1H), 8.19 (d, $J=8.4 \mathrm{~Hz}, 1 \mathrm{H})$, $7.76(\mathrm{~d}, J=8.0 \mathrm{~Hz}, 1 \mathrm{H}), 7.32(\mathrm{~d}, J=7.6 \mathrm{~Hz}, 1 \mathrm{H}), 7.04-$ $7.12(\mathrm{~m}, 1 \mathrm{H}), 6.86-6.93(\mathrm{~m}, 1 \mathrm{H}), 6.83(\mathrm{~d}, J=8.0 \mathrm{~Hz}, 1 \mathrm{H})$, $2.35(\mathrm{~s}, 3 \mathrm{H}) ;{ }^{13} \mathrm{C}$ NMR (100 MHz, DMSO- $\left.d_{6}\right): \delta 169.3$, 156.2 , 155.7, 152.6, 150.3, 141.9, 132.3, 131.2, 130.1, 129.2, 128.9, 125.1, 124.2, 122.3, 121.7, 116.9, 11.5; IR (KBr) $v_{\max } 3276,3208,3073,2956,1646,1485,1368$, 1224, 1146; EI-MS: m/z $323.1(\mathrm{M}-\mathrm{H})^{+}$.

4-(2-Hydroxyphenyl)-6-(trifluoromethyl)quinoline-3carboxylic acid (3k)

White solid; ${ }^{1} \mathrm{H}$ NMR (400 MHz, DMSO- $d_{6}$ ): $\delta 11.4$ (s, $1 \mathrm{H}), 10.01(\mathrm{~s}, 1 \mathrm{H}), 9.61(\mathrm{~s}, 1 \mathrm{H}), 8.32(\mathrm{~d}, J=8.4 \mathrm{~Hz}, 1 \mathrm{H})$, $8.18(\mathrm{~d}, J=7.6 \mathrm{~Hz}, 1 \mathrm{H}), 7.95(\mathrm{dd}, J=8.4 \mathrm{~Hz}, J=2.0 \mathrm{~Hz}$, $1 \mathrm{H}), 7.32$ (d, $J=7.6 \mathrm{~Hz}, 1 \mathrm{H}), 7.01-7.09$ (m, 1H), 6.85$6.91(\mathrm{~m}, 1 \mathrm{H}), 6.81(\mathrm{~d}, J=8.0 \mathrm{~Hz}, 1 \mathrm{H}) ;{ }^{13} \mathrm{C} \mathrm{NMR}(100$ MHz, DMSO- $\left.d_{6}\right): \delta 169.2,155.7,150.6,150.3,142.4$, $130.9,130.5,130.3,129.1,128.1,127.5,125.2,123.1$, 122.3, 121.9, 120.8, 116.8; IR (KBr) $v_{\max } 3278,3206$, 3074, 2956, 1644, 1483, 1370, 1234, 1148; EI-MS: m/z $332.0(\mathrm{M}-\mathrm{H})^{+}$. 


\section{8-Chloro-4-(2-hydroxyphenyl)-6-(trifluoromethyl)quin- oline-3-carboxylic acid (3I)}

White solid; ${ }^{1} \mathrm{H}$ NMR (400 MHz, DMSO- $\left.d_{6}\right): \delta 11.4$ (s, $1 \mathrm{H}), 10.01(\mathrm{~s}, 1 \mathrm{H}), 9.76(\mathrm{~s}, 1 \mathrm{H}), 8.21(\mathrm{~d}, J=8.8 \mathrm{~Hz}, 1 \mathrm{H})$, $8.12(\mathrm{~d}, J=7.6 \mathrm{~Hz}, 1 \mathrm{H}), 7.32(\mathrm{~d}, J=7.6 \mathrm{~Hz}, 1 \mathrm{H}), 7.01-$ $7.09(\mathrm{~m}, 1 \mathrm{H}), 6.85-6.91(\mathrm{~m}, 1 \mathrm{H}), 6.81(\mathrm{~d}, J=8.0 \mathrm{~Hz}, 1 \mathrm{H})$; ${ }^{13} \mathrm{C}$ NMR (100 MHz, DMSO- $\left.d_{6}\right): \delta 169.5,155.7,151.6$, $146.5,142.4,134.9,130.9,130.5,129.1,128.1,127.9$, 126.8, 126.1, 124.7, 122.2, 12.5, 116.8; IR (KBr) $v_{\max }$ 3276, 3210, 3071, 2958, 1648, 1482, 1366, 1226, 1148; EI-MS: m/z $366.5(\mathrm{M}-\mathrm{H})^{+}$.

Acknowledgments. The authors thank University Grants Commission for the financial assistance under Major Research Project. N.Chandra Sekhar thanks CSIR for the Junior Research Fellowship.

\section{REFERENCES}

1. Nasveld, P.; Kitchener, S. Trans. R. Soc. Trop. Med. Hyg. 2005, 99, 2.

2. Leatham, P. A.; Bird, H. A.; Wright, V.; Seymour, D.; Gordon, A. Eur. J. Rheumatol. Inflamm. 1983, 6, 209.

3. Denny, W. A.; Wilson, W. R.; Ware, D. C.; Atwell, G. J.; Milbank, J. B.; Stevenson, R. J. U.S Patent, 7064117, 2006.

4. Mahamoud, A.; Chevalier, J.; Davin-Regli, A.; Barbe, J. Jean Marie Pages. Curr. Drug Targets. 2006, 7, 843.

5. Muruganantham, N.; Sivakumar, R.; Anbalagan, N.; Gunasekaran, V.; Leonard, J. T. Biol. Pharm. Bull. 2004, 27, 1683.

6. Maguire, M. P.; Sheets, K. R.; McVety, K.; Spada, A. P.; Zilberstein, A. J. Med. Chem. 1994, 37, 2129.

7. (a) Wilson, W. D.; Zhao, M.; Patterson, S. E.; Wydra, R. L.; Janda, L.; Strekowski, L. Med. Chem. Res. 1992, 1, 102.

8. Atwell, G. J.; Baguley, B. C.; Denny, W. A. J. Med. Chem. 1989, 32, 396.

9. Xia, Y.; Yang, Z. Y.; Xia, P.; Bastow, K. F.; Tachibana, Y.; Kuo, S. C.; Hamel, E.; Hackl, T.; Lee, K. H. J. Med. Chem. 1998, 41, 1155.

10. Chen, Y. L.; Chen, I. L.; Tzeng, C. C.; Wang, T. C. Helv. Chim. Acta 2000, 83, 989.

11. Jenekhe, S. A.; Lu, L.; Alam, M. M. Macromolecules 2001, 34, 7315.

12. For a recent review, see: Gilchrist, T. L. J. Chem. Soc., Perkin Trans. 1 2001, 2491.

13. Kouznetsov, V. V.; Mendez, L. Y.; Gomez, C. M. Curr. Org. Chem. 2005, 9(2), 141.

14. Chambers, R. D.; Holling, D.; Sandford, G.; Batsanov, A. S.; Howard, J. A. K. J. Fluorine Chem. 2004, 125, 661.

15. Konig, W. Ber. 1923, 56, 1853.

16. Julia, M. Ann. Chim. (France) 1950, 595.

17. Gagan, J. M.; Lloyd, D. J. Chem. Soc., Chem. Commun.
1967, 1043.

18. Acheson, R. M.; Bolton, R. G. Tetrahedron Lett. 1973, 2827.

19. (a) Schroeder, G.; Luttke, W. Chem. Ber. 1972, 105, 2175.

(b) Palmer, M. H. J. Chem. Soc. 1962, 3645.

20. (a) McNab, H.; Murray, E. A. J. Chem. Soc., Perkin Trans. 1 1988, 333. (b) McNab, H.; Murray, E. A. ARKIVOC 2002, iii, 95.

21. Robert, G. F. J. Comb. Chem. 2000, 1, 195.

22. (a) Matsugi, M.; Tabusa, F.; Minamikawa, J. Tetrahedron Lett. 2000, 41, 8523. (b) Ranu, B. C.; Hajra, A.; Dey, S. S.; Jana, U. Tetrahedron 2003, 59, 813. (c) For the latest leading reference on the mechanism of Skraup-DoebnerVon Miller quinoline synthesis, see: Denmark, S. E.; Venkatraman, S. J. Org. Chem. 2006, 71, 1668.

23. Wu, Y.-C.; Li, L.; Li, H.-J.; Wang, D.; Chen, Y.-J. J. Org. Chem. 2006, 71, 6592.

24. Tanaka, S.-Y.; Yasuda, M.; Baba, A. J. Org. Chem. 2006, 71,800 .

25. Dieter Heber Arch. Pharma. (Weinheim) 1987, 320, 595.

26. (a) Matsugi, M.; Tabusa, F.; Minamikawa, J.-I. Tetrahedron Lett. 2000, 41, 8523. (b) Panda, K.; Siddiqui, I.; Mahata, P. K.; Ila, H.; Junjappa, H. Synlett 2004, 449.

27. More than three step reaction sequences were taken for quinoline synthesis. See: (a) Ishikawa, T.; Manabe, S.; Aikawa, T.; Kudo, T.; Saito, S. Org. Lett. 2004, 6, 2361. (b) Theeraladanon, C.; Arisawa, M.; Nishida, A.; Nakagawa, M. Tetrahedron 2004, 60, 3017. (c) Ichikawa, J.; Wada, Y.; Miyazaki, H.; Mori, T.; Kuroki, H. Org. Lett. 2003, 5, 1455.

28. (a) Amii, H.; Kishikawa, Y.; Uneyama, K. Org. Lett. 2001, 3, 1109. (b) Zhao, F.; yang, X.; Liu, J. Tetrahedron 2004, 60, 9945. (c) Cho, C. S.; Kim, B. T.; Kim, T.-J.; Shim, S. C. Chem. Commun. 2001, 2576. (d) Takashi, M.; Ichikawa, J. Chem. Lett. 2004, 33, 590.

29. (a) Jiang, B.; Si, Y.-G. J. Org. Chem. 2002, 67, 9449. (b) Cho, C. S.; Kim, T. K.; Kim, B. T.; Kim, T.-J.; Shim, S. C. J. Organomet. Chem. 2002, 650, 65. (c) McNaughton, B. R.; Miller, B. L. Org. Lett. 2003, 5, 4257. (d) Du, W.; Curran, D. P. Org. Lett. 2003, 5, 1765.

30. (a) Akiyama, T.; Nakashima, S.; Yokota, K.; Fuchibe, K. Chem. Lett. 2004, 33, 922. (b) Cho, C. S.; Oh, B. H.; Kim, J. S.; Kim, T.-J.; Shim, S. C. Chem. Commun. 2000, 1885. (c) Sangu, K.; Fuchibe, K.; Akiyama, T. Org. Lett. 2004, 6, 353.

31. Mahata, P. K.; Venkatesh, C.; Kumar, U. K. S.; Ila, H.; Junjappa, H. J. Org. Chem. 2003, 68, 3966. Most other quinoline syntheses using Vilsmeier-type reactions referenced therein also need more than an equimolar amount of $\mathrm{POCl}_{3}$.

32. Recently, some useful approaches assisted by microwave and/or Lewis acid catalysts have been reported, although none of them were performed on a large scale. (a) Yadav, J. S.; Reddy, B. V. S.; Rao, R. S.; Naveenkumar, V.; Nagaiah, K. Synthesis 2003, 1610. (b) Yadav, J. S.; Reddy, B. V. S.; Premalatha, K. Synlett 2004, 963. (c) Yadav, J. S.; 
Reddy, B. V. S.; Sreedhar, R.; Rao, S.; Nagaiah, K. Synthesis 2004, 14, 2381. (d) De, S. K.; Gibbs, R. A. Tetrahedron Lett. 2005, 46, 1647.

33. (a) Zhang, X.; Yao, T.; Campo, M.A.; Larock, R.C. Tetra- hedron Lett. 2010, 66, 1177. (b) Subhas, B.; Idrees, D.; Jakka, M.; Venkateswara Rao, N. M. J. J. Comb. Chem. 2010, 12, 100. (c) Shan, G.; Sun, X.; Xia, Q.; Rao, Y. Org. Lett. 2011, 10.1021/ol202334s. 